http://dx.doi.org/10.1590/1678-4162-9303

Arq. Bras. Med. Vet. Zootec., v.69, n.5, p.1186-1190, 2017

\title{
Bloqueio de plexo braquial em um bugio-ruivo (Alouatta guariba): relato de caso
}

[Brachial plexus block in a red howler monkey (Alouatta guariba): case report]

\author{
E.R. Santos ${ }^{1}$, B.S. Barni ${ }^{1}$, L.A.F. Colombi ${ }^{1}$,C.S. Braga ${ }^{1}$, V.S. Mombach ${ }^{2}$, \\ M.S. Muccillo ${ }^{1}$, M.M. Alievi ${ }^{3}$, E.A. Contesini ${ }^{3}$
}

\footnotetext{
${ }^{1}$ Hospital de Clínicas Veterinárias - Universidade Federal do Rio Grande do Sul - Porto Alegre, RS ${ }^{2}$ Programa de pós-graduação - Universidade Federal do Rio Grande do Sul - Porto Alegre, RS

${ }^{3}$ Faculdade de Veterinária - Universidade Federal do Rio Grande do Sul - Porto Alegre, RS
}

\begin{abstract}
RESUMO
Os bloqueios regionais vêm sendo explorados e difundidos no dia a dia da medicina veterinária. $\mathrm{O}$ presente trabalho relata a execução do bloqueio de plexo braquial em um bugio-ruivo (Alouatta guariba) submetido à amputação de três dígitos. Foi utilizada ropivacaína para o bloqueio, com o paciente sob anestesia geral, com auxílio de neuroestimulador periférico. Quatro horas após a cirurgia e cinco horas após o bloqueio, o paciente não apresentava desconforto ou evitava a manipulação do membro operado e não foram observadas complicações. O bloqueio do plexo braquial é mais comumente utilizado quando se pretende promover analgesia e relaxamento muscular do membro torácico em procedimentos cirúrgicos distais à articulação escápulo-umeral. Há carência em estudos anatômicos sobre diversos primatas, incluindo o bugio-ruivo (Alouatta guariba). Apesar da crescente expansão da clínica de animais silvestres, ainda há lacunas no que diz respeito à execução de técnicas anestésicas em diversas espécies.
\end{abstract}

Palavras-chave: anestesia, analgesia, primata, animais silvestres

\begin{abstract}
The regional blocks are being exploited and disseminated on a daily basis of veterinary medicine. This study describes the implementation of the brachial plexus block in a red howler-monkey (Alouatta guariba) that underwent amputation of three digits. Ropivacaine was employed for blocking, with the patient under general anesthesia, with the aid of a peripheral nerve stimulator. Four hours after surgery, and five hours after the blockade, the patient had no discomfort nor avoided manipulation of the operated limb and no complications were observed. The brachial plexus block is most commonly used when you want to promote analgesia and muscle relaxation of the forelimb in distal surgical procedures to the scapular-humeral joint. There is a lack of anatomical studies on various primates, including the red howler-monkey (Alouatta guariba). Despite the growing expansion of clinic of wild animals, there are still shortcomings with regards to the implementation of anesthetic techniques in many species.
\end{abstract}

Keywords:anesthesia, analgesia, primate, wild animals

\section{INTRODUÇÃO}

Na medicina veterinária, os bloqueios regionais vêm sendo mais explorados e difundidos a cada dia, com estudos e aperfeiçoamentos nas técnicas a serem realizadas. O bloqueio do plexo braquial é mais comumente utilizado quando se pretende promover analgesia e contribuir para o relaxamento muscular do membro torácico para realizações de procedimentos cirúrgicos distais à articulação escápulo-umeral (Klaumann e Otero, 2013).

O bugio-ruivo (Alouatta guariba) é um primata da família Atelidae e do gênero Alouatta. Esse gênero apresenta, entre os primatas, ampla distribuição geográfica no Novo Mundo (Platirrinos), sendo encontrado do México à Argentina. O A. guariba distribui-se desde o sul

Recebido em 26 de setembro de 2016

Aceito em 10 de outubro de 2016

E-mail: eduardo.rosasantos@gmail.com 
da Bahia até o norte da Argentina, e é uma das três espécies de primatas encontradas no Rio Grande do Sul (Chagas et al., 2010).

Há escassez de estudos anatômicos sobre diversos primatas, incluindo a espécie em questão. No entanto, sabe-se que o plexo braquial do macaco-de-cheiro (Saimiri sciureus) é constituído por fibras neurais provenientes da união das raízes dorsais e ventrais das vértebras C4 a C8 e T1, sendo organizado em quatro troncos. Os nervos originados desses quatro troncos possuem origem e trajetória semelhantes em outros primatas, com pequenas variações (Araújo et al., 2012).

$\mathrm{Na}$ espécie macaco-barrigudo (Lagothrix lagothricha), o plexo braquial origina-se dos nervos espinhais C5 a T1, constituindo os troncos cranial, médio e caudal. Os nervos periféricos derivados dos troncos assemelham-se na origem, no trajeto e no território de inervação com os plexos de outros primatas, com exceção do nervo musculocutâneo, que atravessa o músculo coracobraquial. $\mathrm{Na}$ formação dos nervos do plexo, há a participação de um, dois ou três troncos nervosos (Cruz e Adami, 2010).

Apesar da crescente expansão da clínica de animais silvestres, ainda há lacunas no que diz respeito à execução de técnicas anestésicas em diversas espécies. Assim, este trabalho tem por objetivo relatar a execução de um bloqueio de plexo braquial em um bugio-ruivo submetido à amputação de três dígitos.

\section{CASUÍSTICA}

Foi atendido, no Núcleo de Conservação e Reabilitação de Animais Silvestres do Hospital de Clínicas Veterinárias da Universidade Federal do Rio Grande do Sul (Preservas/HCV/UFRGS), um bugio-ruivo (Alouatta guariba) encontrado na região rural de Porto Alegre. O paciente apresentava lesões características de mordedura em três dígitos do membro torácico esquerdo. Realizou-se coleta de sangue para hemograma e perfil bioquímico. As alterações verificadas foram discreta anemia, leucocitose por neutrofilia, eosinofilia, monocitose e linfopenia.
O paciente permaneceu internado, recebendo tratamento de suporte. Após duas semanas de tratamento, a lesão não apresentou melhora significativa e optou-se, então, pela amputação cirúrgica dos dígitos acometidos.

A medicação pré-anestésica (MPA) foi composta por cloridrato de cetamina $\left(8 \mathrm{mg} . \mathrm{kg}^{-1}\right)$ e maleato de midazolam $\left(0,3 \mathrm{mg} \cdot \mathrm{kg}^{-1}\right)$, ambos pela via intramuscular (IM). Em seguida, foi realizada pré-oxigenção através de máscara de oxigênio, durante cinco minutos. Efetuou-se acesso vascular da veia axilar para a indução anestésica com propofol $1 \%\left(2 \mathrm{mg} \cdot \mathrm{kg}^{-1}\right)$, ocasionando a perda do reflexo palpebral medial e o relaxamento do tônus mandibular. O paciente foi posicionado em decúbito dorsal, intubado com sonda endotraqueal $n^{\circ}$ 4,5 e mantido em sistema de anestesia aberto, com fluxo de oxigênio de $2 \mathrm{~L}$ e isoflurano ao efeito em vaporizador universal. O monitoramento foi realizado com eletrocardiograma, oximetria de pulso, pressão arterial não invasiva e capnografia durante toda a anestesia.

Em seguida, para o bloqueio do plexo braquial, posicionou-se o paciente em decúbito lateral esquerdo e efetuou-se antissepsia da região e palpação para localização das referências anatômicas para o bloqueio. As musculaturas subclávia e peitoral foram localizadas, e introduziu-se entre elas a agulha (Stimuplex 22G, B Braun) levemente lateralizada para a direção da escápula, iniciando a estimulação com neuroestimulador periférico (Stimuplex HNS 12, B Braun) a 1,5 miliampère (mA) (Fig. 1). A agulha foi angulada até a obtenção da resposta motora de contração do músculo bíceps braquial e leve flexão do cotovelo, em menor estimulação, que foi de $0,5 \mathrm{~mA}$. Injetaram-se $2 \mathrm{~mL}$ de ropivacaína $\left(7,5 \mathrm{mg} \cdot \mathrm{mL}^{-1}\right)$, após certificar-se de que a agulha não estava localizada no interior de um vaso sanguíneo. Observou-se a parada do estímulo, confirmando a exata localização do plexo. No momento da injeção do anestésico local, a impedância foi de 58,5 quilo-ohm $(\mathrm{k} \Omega)$, a qual variou de 32 até $69,2 \mathrm{k} \Omega$. 


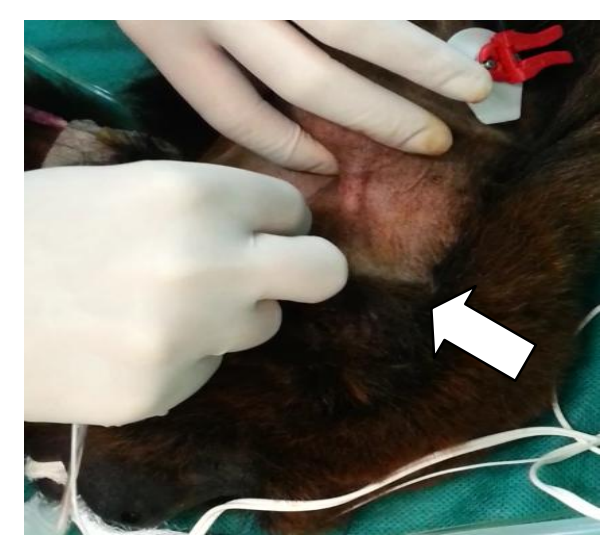

Figura 1. Inserção da agulha (seta) entre musculaturas subclávia e peitoral para neuroestimulação periférica.

Procedeu-se à cirurgia de amputação do primeiro, segundo e terceiro dígitos. Durante o procedimento, não ocorreram variações significativas nos parâmetros avaliados, sugerindo eficácia do bloqueio do membro (Tab. 1). Ao término do procedimento, o paciente despertou rapidamente e sem agitação.

Tabela 1. Parâmetros fisiológicos avaliados durante procedimento anestésico e cirúrgico de amputação de dígitos em bugio-ruivo (Alouatta guariba)

\begin{tabular}{lccccccc}
\hline Tempo $(\mathrm{min})$ & 0 & 10 & 20 & 30 & 40 & 50 & 60 \\
\hline FC $(\mathrm{bpm})$ & 130 & 115 & 125 & 133 & 132 & 128 & 122 \\
$\mathrm{FR}(\mathrm{mpm})$ & 10 & 10 & 10 & 13 & 14 & 15 & 16 \\
$\mathrm{SpO}_{2}(\%)$ & 98 & 95 & 96 & 98 & 98 & 94 & 96 \\
$\mathrm{EtCO}_{2}(\%)$ & 40 & 42 & 42 & 49 & 49 & 49 & 47 \\
$\mathrm{PAS}(\mathrm{mmHg})$ & - & - & 95 & 89 & 85 & 86 & 87 \\
$\mathrm{PAM}(\mathrm{mmHg})$ & - & - & 70 & 65 & 60 & 64 & 64 \\
$\mathrm{PAD}(\mathrm{mmHg})$ & - & - & 57 & 43 & 50 & 49 & 55 \\
$\mathrm{~T}\left({ }^{\circ} \mathrm{C}\right)$ & 37 & 37 & 36,1 & 36 & 35,4 & 33,8 & 36,4 \\
\hline
\end{tabular}

Com cerca de quatro horas de pós-operatório e cinco horas após o bloqueio, o paciente se apresentava em estação, alimentando-se normalmente, sem apresentar resistência ou desconforto à manipulação do membro operado. Ao pinçamento dos dígitos restantes, não esboçou reação, sugerindo que os bloqueios motor e sensitivo ainda estavam presentes.

Como terapia pós-operatória, iniciada após quatro horas do procedimento, utilizou-se o cloridrato de tramadol $\left(5 \mathrm{mg} \cdot \mathrm{kg}^{-1}, \quad \mathrm{BID}\right.$, subcutâneo, por cinco dias), amoxicilina com ácido clavulânico (20mg. $\mathrm{kg}^{-1}, \quad$ SID, intramuscular, por 10 dias), dipirona sódica (25mg.kg ${ }^{-1}$, oral, BID, por sete dias) e carprofeno (4mg. $\mathrm{kg}^{-1}, \mathrm{SID}$, subcutâneo, por três dias).

\section{DISCUSSÃO}

No presente trabalho, a MPA foi importante e eficaz para a contenção do paciente antes da manipulação, minimizando o estresse, conforme Chagas et al. (2010) afirmaram.

Utilizou-se cloridrato de cetamina como um dos fármacos da MPA, o qual, segundo Vasconcellos et al. (2000), é a droga mais utilizada para contenção química, sedação ou anestesia de primatas não humanos. Além disso, apresenta vantagens que puderam ser confirmadas no presente caso, como elevado índice terapêutico, rápido início de ação e curta duração.

O maleato de midazolam, associado ao cloridrato de cetamina, promoveu um adequado miorrelaxamento, reduzindo, assim, a hipertonicidade muscular causada pela cetamina, corroborando Vasconcellos et al. (2000). Chagas et al. (2010) citaram que o midazolam é uma boa 
escolha, pois ainda promove tranquilização, hipnose, amnésia, além de possuir atividade anticonvulsivante. Por apresentar veículo aquoso, pode ser aplicado pela via intramuscular, apresentando rápida absorção e eliminação, vantagens confirmadas no paciente do estudo.

A utilização do propofol se mostrou uma boa opção, com rápida indução e recuperação, concordando com Capriglione et al. (2013), que destacaram o fármaco em virtude dessas características, além de ser rapidamente metabolizado.

O anestésico inalatório isoflurano forneceu estabilidade e segurança durante o procedimento, mostrando-se adequado para a espécie, assim como sustentado por Vasconcellos et al. (2000), os quais afirmam que agentes inalatórios são indicados para manutenção anestésica de primatas, previamente contidos com fármacos injetáveis, administrados por máscara ou sonda endotraqueal.

As referências utilizadas para a abordagem do plexo braquial no bugio-ruivo foram semelhantes às utilizadas em humanos, com exceção do decúbito, que, neste caso, foi o lateral. Segundo Muñoz et al. (2010), em humanos, a abordagem se dá em decúbito dorsal, com a cabeça lateralizada ao contrário do lado a ser bloqueado. O local a ser puncionado é a borda interna da cabeça clavicular, no músculo esternocleidomastoideo, a $3 \mathrm{~cm}$ de altura do nível do esterno.

A utilização do cloridrato de ropivacaína no presente caso demonstrou efeito dentro do esperado, com analgesia duradoura, sendo eficiente para o bloqueio em questão, concordando, assim, com Wakoff et al. (2013), que descrevem a ropivacaína como um anestésico local de longa duração, possuindo menos efeitos colaterais cardiovasculares e neurológicos do que outros anestésicos locais comumente utilizados.

Os estímulos encontrados inicialmente na frequência de $1,5 \mathrm{~mA}$ e cessados em $0,5 \mathrm{~mA}$ corroboram o que descreveram Klaumann e Otero (2013), os quais referiram que estimulações nervosas com correntes superiores a $0,2 \mathrm{~mA}$ e menores ou iguais a $0,5 \mathrm{~mA}$ são associadas a bloqueios anestésicos eficientes. A contração do músculo bíceps braquial e a leve flexão do cotovelo encontradas na estimulação de $0,5 \mathrm{~mA}$ e cessadas ao injetar a solução anestésica também vão ao encontro do que os mesmos autores citaram como estímulos padrão na neuroestimulação do plexo braquial.

No momento em que se encontrou o melhor estímulo, a injeção de anestésico local foi aplicada, e a impedância era de $58,5 \mathrm{k} \Omega$, o que sugere uma proximidade perineural. Apesar disso, clinicamente o bloqueio foi efetivo. Segundo Tsui et al. (2008), a variação da impedância nos tecidos se deve à heterogenicidade deles, principalmente devido à variação no teor de água e lipídios. O nervo periférico é uma estrutura complexa, que consiste em fascículos (cada feixe de fibra nervosa cercada por um perineuro), os quais são mantidos juntos pelo epineuro, uma bainha de tecido conjuntivo externo que os envolve (Tsui et al., 2008). Vários tecidos fibrosos dentro do compartimento intraneural contêm maiores quantidades de lipídios não condutores, fluido intersticial e menor teor de água do que os músculos circundantes. Portanto, a mudança abrupta na impedância que ocorre ao entrar em um nervo a partir de um tecido circundante é facilmente detectada (Tsui et al., 2008), o que sugere que a variação apresentada de 32 a $69,2 \mathrm{k} \Omega$ foi decorrente da mudança tecidual em contato com a ponta da agulha, podendo ser o tecido fibroso ou a proximidade com o compartimento intraneural.

Não foram observadas no animal quaisquer complicações relacionadas ao bloqueio do plexo braquial, embora estas possam ocorrer em alguns casos. Segundo Adami e Studer (2015), pode ser observada arritmia ventricular severa devido à introdução inadvertida da agulha na cavidade torácica. Ainda, Ridge (2014) observou efeito prolongado do anestésico local, entretanto, no caso por ele relatado, não foi utilizado $o$ neuroestimulador periférico, o que tornou $\mathrm{o}$ procedimento mais arriscado.

\section{CONCLUSÃO}

Apesar da escassez de referências anatômicas em primatas, a extrapolação da anatomia humana foi eficaz para a realização correta do bloqueio do plexo braquial do bugio-ruivo (Alouatta guariba), por meio de neuroestimulador 
periférico, fornecendo adequada analgesia trans e pós-operatória e ausência de complicações.

\section{REFERÊNCIAS}

ADAMI, C.; STUDER, N. A case of severe ventricular arrhythmias occurring as a complication of nerve-stimulator guided brachial plexus location. Vet. Anaesth. Analg., v.42, p.230-231, 2015.

ARAÚJO, E.B.; LIMA, A.R.; PINHEIRO, L.L. et al. Origem e distribuição do plexo braquial de Saimiri sciureus. Pesqui. Vet. Bras., v.32, p.1351-1354, 2012.

CAPRIGLIONE， L.G.A.; SORESINI， G.C.; FUCHS, T. et al. Avaliação eletrocardiográfica de macacos-prego (Sapajus apella) sob contenção química com midazolam e propofol. Semin. Ciênc. Agrár. v.34, Supl.2, p.3801-3810, 2013.

CHAGAS, J.A.B.; OLESKOVICZ, N.; MORAES, A.N.D. Associação de cetamina S(+) e midazolam pelo método convencional de cálculo e pela extrapolação alométrica em bugios-ruivo (Alouatta guariba clamitans): resposta clínica e cardiorrespiratória. Cienc. Rural, v.40, p.109-104, 2010.

CRUZ, G.A.M.; ADAMI, M. Anatomia do plexo braquial de macaco-barrigudo (Lagothrix lagothricha). Pesqui. Vet. Bras., v.30, p.881-886, 2010.
KLAUMANN, P.R.; OTERO, P.E. Anestesia locorregional em pequenos animais. São Paulo: ROCA, 2013. 288p.

MUÑOZ, C.M.; GONZÁLEZ, R.O.T.; NAVAS, A.M. et al. Estudio comparativo de la eficácia del bloqueo supraclavicular em la artroscopia de hombro. Rev. Soc. Esp. Dolor, v.17, p.366-371, 2010.

RIDGE, P. Complication following a brachial plexus block. Vet. Rec., v.174, p.614, 2014.

TSUI, C.B.; PILLARY, J.J.; CHU, K.T. et al. Electrical impedance to distinguish intaneural from extraneural needle placement in porcine nerves during direct exposure and ultrasound guidance. Anesthesiology, v.109, p.479-483, 2008.

VASCONCELLOS, C.H.C.; MÁRSICO FILHO, F.; SEGURA, I.A.G. et al. Utilização do isoflurano em macacos-prego (Cebus apella). Braz. J. Vet. Rev. Anim. Sci., v.37, p.65-69, 2000.

WAKOFF, T.I.; MENCALHA, R.; SOUZA, N.S. et al. Bupivacaína $0,25 \%$ versus ropivacaína $0,25 \%$ no bloqueio de plexo braquial em cães da raça beagle. Semin. Ciênc. Agrár., v.34, p.12591272, 2013. 